\title{
Europäische Pressemärkte - Grundlagen und Statistik des Zeitungswesens
}

\section{Dieter Stürzebecher}

Sich mit einer Statistik des Zeitungswesens oder - weiter gefasst - mit Pressestatistik zu beschäftigen, ist weder Selbstzweck noch Hobby. Der Bedarf an einer fundierten und aussagefähigen Statistik des Zeitungswesens ergibt sich vielmehr aus der öffentlichen Aufgabe und den herausragenden politischen Funktionen - wie übrigens auch aus den vielfältigen sonstigen, nicht-politischen Funktionen -, die das ältestes Massenmedium der Welt in allen entwickelten, demokratischen Gesellschaften erfüllt bzw. zu erfüllen hat. Im Idealfall können und sollten die Befunde einer Zeitungsstatistik medienpolitisches oder wettbewerbsrechtliches Handeln zur Sicherstellung dieser öffentlichen Aufgabe beeinflussen - oder es überhaupt erst initiieren. Das war in Deutschland insbesondere in den 70er-Jahren der Fall, als die Fakten aus eben jener Zeitungsstatistik eine ausgesprochen dynamisch fortschreitende Konzentration auf dem Pressemarkt dokumentierten - und ein medienpolitisches Gegensteuern geradezu aufdrängten.

Eine Pressestatistik - und die Ergebnisse und Daten, die sie zutage fördert - kann allerdings nur so aussagefähig sein wie die ihr zugrunde liegenden Kriterien und Kategorien

- eindeutig und präzise definiert sind,

- sich als geeignet und praktikabel erweisen, um die Strukturen und Besonderheiten auf dem Pressemarkt transparent zu machen, und schließlich

- operationalisierbar, also anwendbar in dem Sinne sind, dass sie sich mit verlässlichen Zahlen füllen und fundieren lassen.

Die Definition des Pressetyps „Zeitung“ und die statistischen Kategorien, die in Deutschland zur Beschreibung und Analyse des Zeitungsmarktes verwendet werden, haben sich nun in der Tat als sehr leistungsfähig erwiesen. Damit war es möglich, statistische Ergebnisse - insbesondere auch im Zeitverlauf mit einem sehr hohen Informationsgehalt zu generieren.

Doch können sie auch den Erfordernissen einer vergleichenden europäischen Zeitungsstatistik gerecht werden? Bei dem ambitionierten Vorhaben einer europäischen Statistik des Zeitungswesens wurden in einem ersten Anlauf im Wesentlichen auf jenen hierzulande entwickelten und bewährten Kategorien, Kriterien und Definitionen aufgebaut. Eine der wesentlichen Aufgaben war es 
im weiteren Verlauf, diese Definitionen und statistischen Kategorien auf deren Internationalität hin zu testen. Im Mittelpunkt standen dabei zwei Überlegungen:

1. Sind die vorgeschlagenen Kategorien übertragbar und geeignet, um die wesentlichen Merkmale und Besonderheiten der Zeitungsmärkte anderer Länder, im Idealfall sogar aller europäischen Länder präzise abbilden zu können? Anders formuliert: Brauchen wir womöglich eine Modifikation oder gar einen ganz anderen Zuschnitt der statistischen Maßeinheiten, weil nämlich jene, die zunächst einmal vorgeschlagen wurden, sich viel zu stark an Strukturen des Zeitungswesens orientieren, die es womöglich nur in Deutschland, aber in keinem anderen Land gibt?

2. Sind die in Deutschland entwickelten statistischen Kategorien im Hinblick auf eine europäische Zeitungsstatistik womöglich auch deshalb zu überdenken, weil sie andernfalls nicht lösbare Datenzugangs- und Datenbeschaffungsprobleme mit sich bringen würden? Auch dies anders formuliert: Was nützen die besten statistischen Kategorien und Maßeinheiten, wenn die Daten und Fakten, die damit gewonnen werden sollen, mit vertretbarem Aufwand gar nicht zu beschaffen sind?

Vor allem diese beiden Gesichtpunkte waren der Dreh- und Angelpunkt bei der Frage nach der Praktikabilität von Definitionen und Kategorien für eine europäische Statistik des Zeitungswesens.

Ausgangspunkt und Grundlage der hierzulande bewährten Zeitungsstatistik ist eine relativ restriktive Definition des Begriffs „Zeitung“. Diese Definition fordert als essentielle Eigenschaften einer Zeitung

- deren öffentliche Verfügbarkeit,

- die Dauerhaftigkeit ihres Erscheinens,

- eine regelmäßige, mindestens zweimal wöchentliche und damit aktualitätsbezogene Erscheinungsweise,

- eine prinzipiell unbegrenzte Themenvielfalt,

- das Bestreben, die größtmögliche Aktualität in der Berichterstattung zu gewährleisten, sowie

- deren Auslieferung als fertig gedrucktes Produkt.

Die maßgebenden Kriterien sind also: Publizität, Kontinuität, Periodizität, Universalität, primäre Aktualität und Druck vor Vertrieb. Als Zeitung gelten dabei ausschließlich solche Presseprodukte, die alle diese Kriterien erfüllen. Ausgeschlossen werden somit Presseprodukte, die zwar äußerlich einer Zeitung gleichen oder der Eigenbezeichnung nach sich dem Publikum als „Zeitung“ präsentieren mögen, die jedoch nicht die umfassende Funktion eines tagesaktuellen und thematisch universellen Periodikums erfüllen. Allerdings wurden solche zeitungsähnlichen Produkte im Zuge der Erhebung an gesonderter Stelle mit erfasst. 
Hat sich nun dieser Zeitungsbegriff als brauchbar für eine europäische Zeitungsstatistik erwiesen? Die Ergebnisse - und ebenso die Ergebnislücken aus den untersuchten Ländern wurden in einer Synopse einander gegenübergestellt, die sich auf eine erste kritische Durchsicht und Analyse der ursprünglich von den beteiligten Wissenschaftlern eingereichten Erhebungsbögen stützt und die als Grundlage für die Diskussionen während der Tagung im Januar 2003 in Hannover und für eine Überarbeitung der Daten diente. Diese vergleichende Übersicht bietet ein gemischtes, teilweise kontrastreiches und zum Teil widersprüchliches Bild: Die Datenbasis und Datengüte ist insgesamt sehr heterogen; die relevanten Kategorien und Maßeinheiten, für die die beteiligten Wissenschaftler Daten gesammelt oder zu sammeln versucht haben, besitzen offenkundig nicht immer und nicht überall den passenden Zuschnitt oder die erforderliche Tragfähigkeit, um damit eine vergleichende, weitgehend homogene Statistik des europäischen Zeitungswesens aufzubauen. Eine zusammenfassende Analyse und Bewertung der Praktikabilität der zugrunde gelegten Kategorien und der Datenlage in den beteiligten Ländern macht dieses Dilemma deutlich.

Einige der Widersprüche und Zweifelsfragen konnten im Rahmen der Tagungsdiskussionen und durch Recherchen in der Folgezeit geklärt werden. Bewertungen, die daher inzwischen überholt oder nicht mehr relevant sind insbesondere durch die Angaben in der vorangehenden Tabelle der Länderergebnisse und durch die in Teil 3 abgedruckten Länderberichte - sind daher in den der Synopse folgenden Länderprofilen mit spitzen Klammern <> markiert. 


\begin{tabular}{|c|c|c|c|c|c|c|c|}
\hline 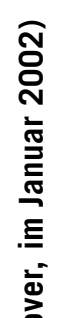 & & 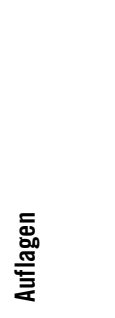 & 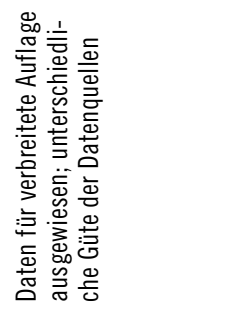 & 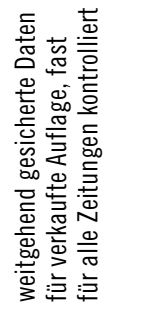 & 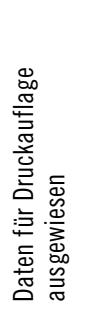 & 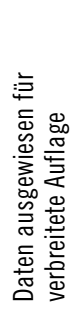 & 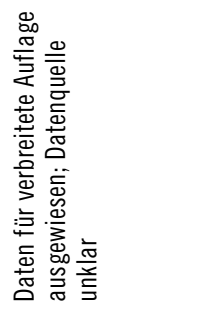 \\
\hline 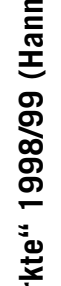 & & 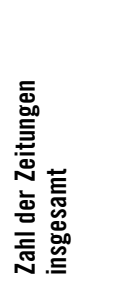 & 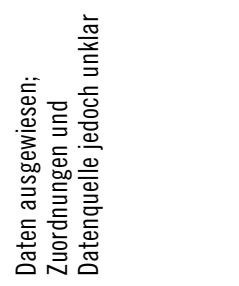 & 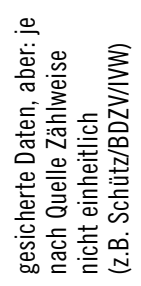 & 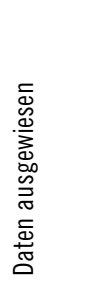 & 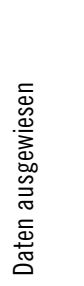 & 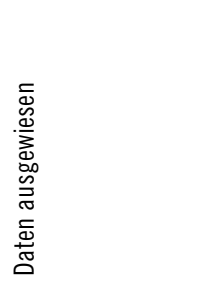 \\
\hline 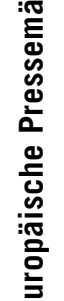 & & 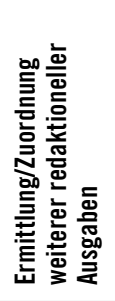 & 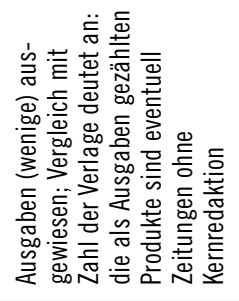 & 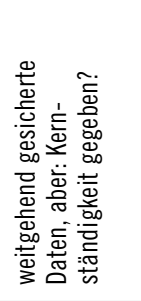 & 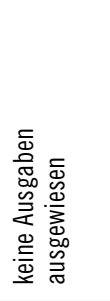 & 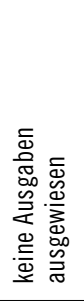 & 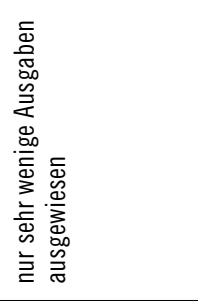 \\
\hline 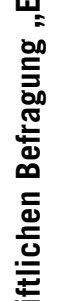 & & 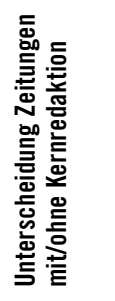 & 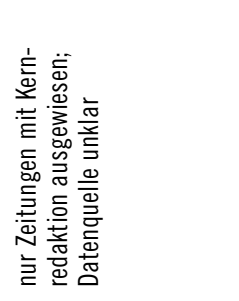 & 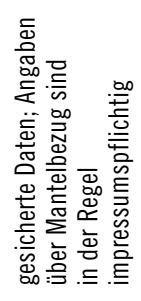 & 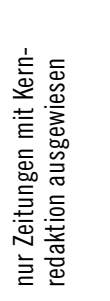 & 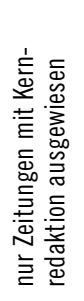 & 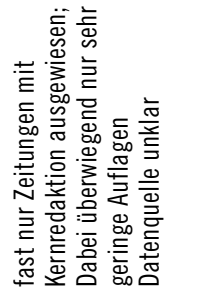 \\
\hline 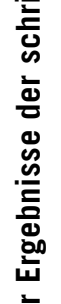 & 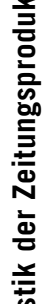 & 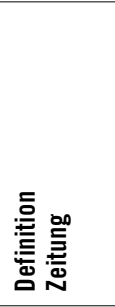 & 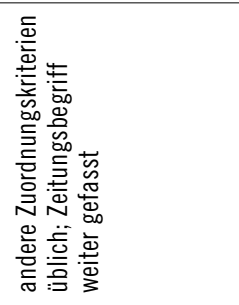 & 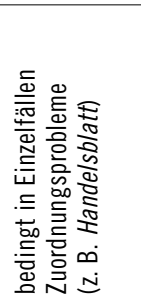 & 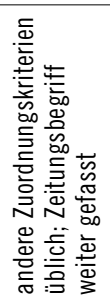 & 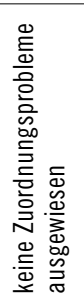 & 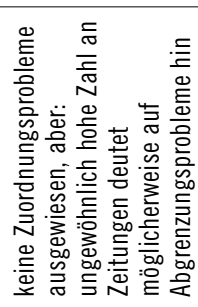 \\
\hline 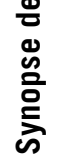 & 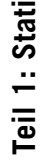 & & $\begin{array}{l}\text { 흘 } \\
\text { 흥 } \\
\overline{\bar{D}}\end{array}$ & 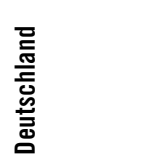 & 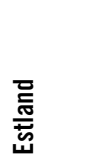 & $\begin{array}{l}\text { 믐 } \\
\text { 뜨 }\end{array}$ & 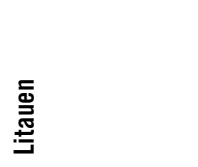 \\
\hline
\end{tabular}




\begin{tabular}{|c|c|c|c|c|c|}
\hline 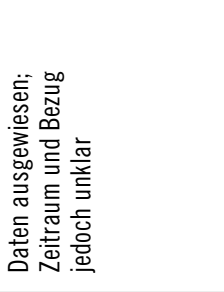 & 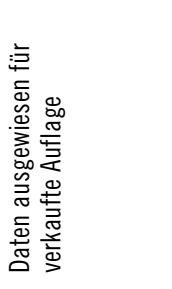 & 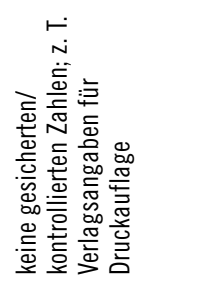 & 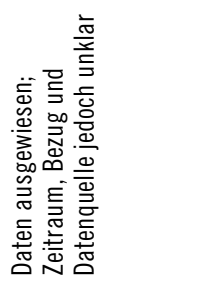 & 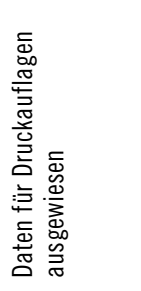 & 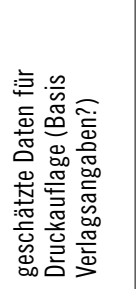 \\
\hline 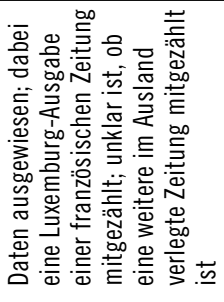 & 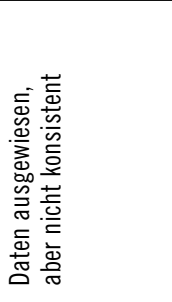 & 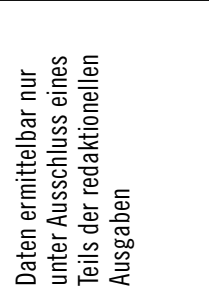 & 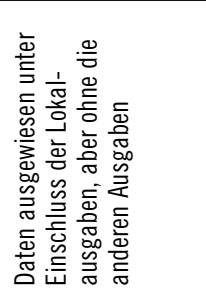 & 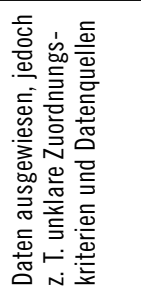 & 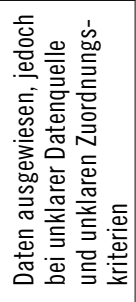 \\
\hline 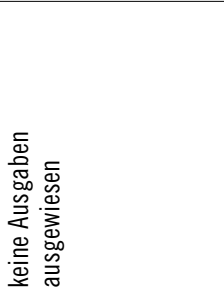 & 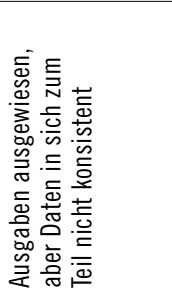 & 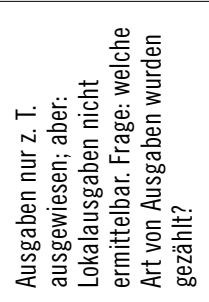 & 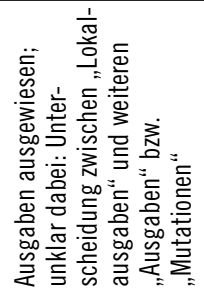 & 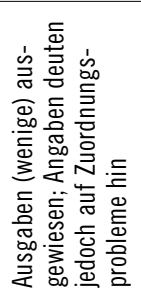 & 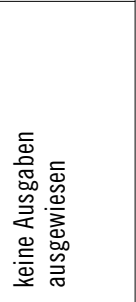 \\
\hline 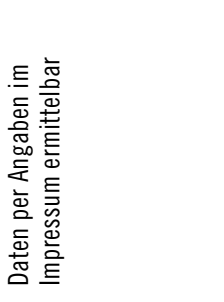 & 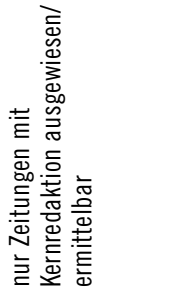 & 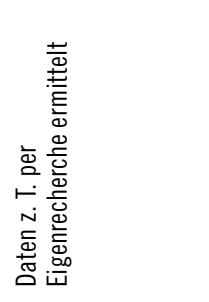 & 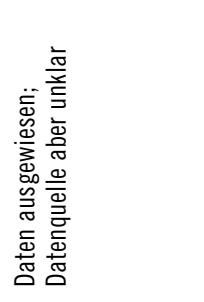 & 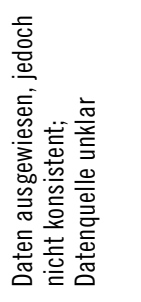 & 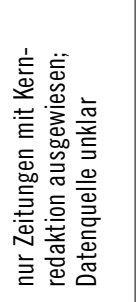 \\
\hline 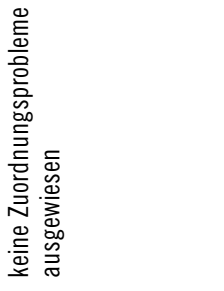 & 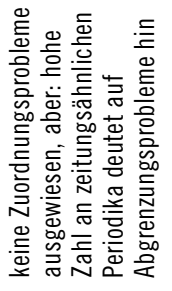 & 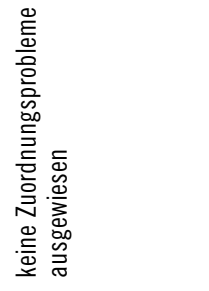 & 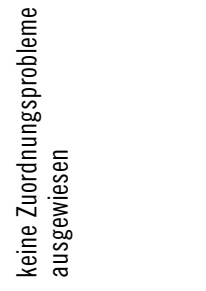 & 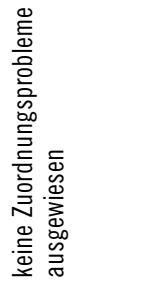 & 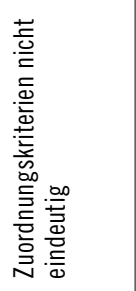 \\
\hline 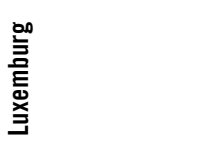 & 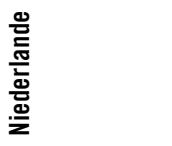 & 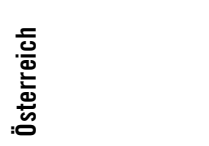 & $\frac{\bar{\sigma}}{0}$ & 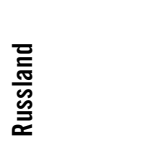 & 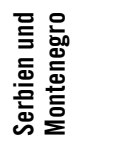 \\
\hline
\end{tabular}




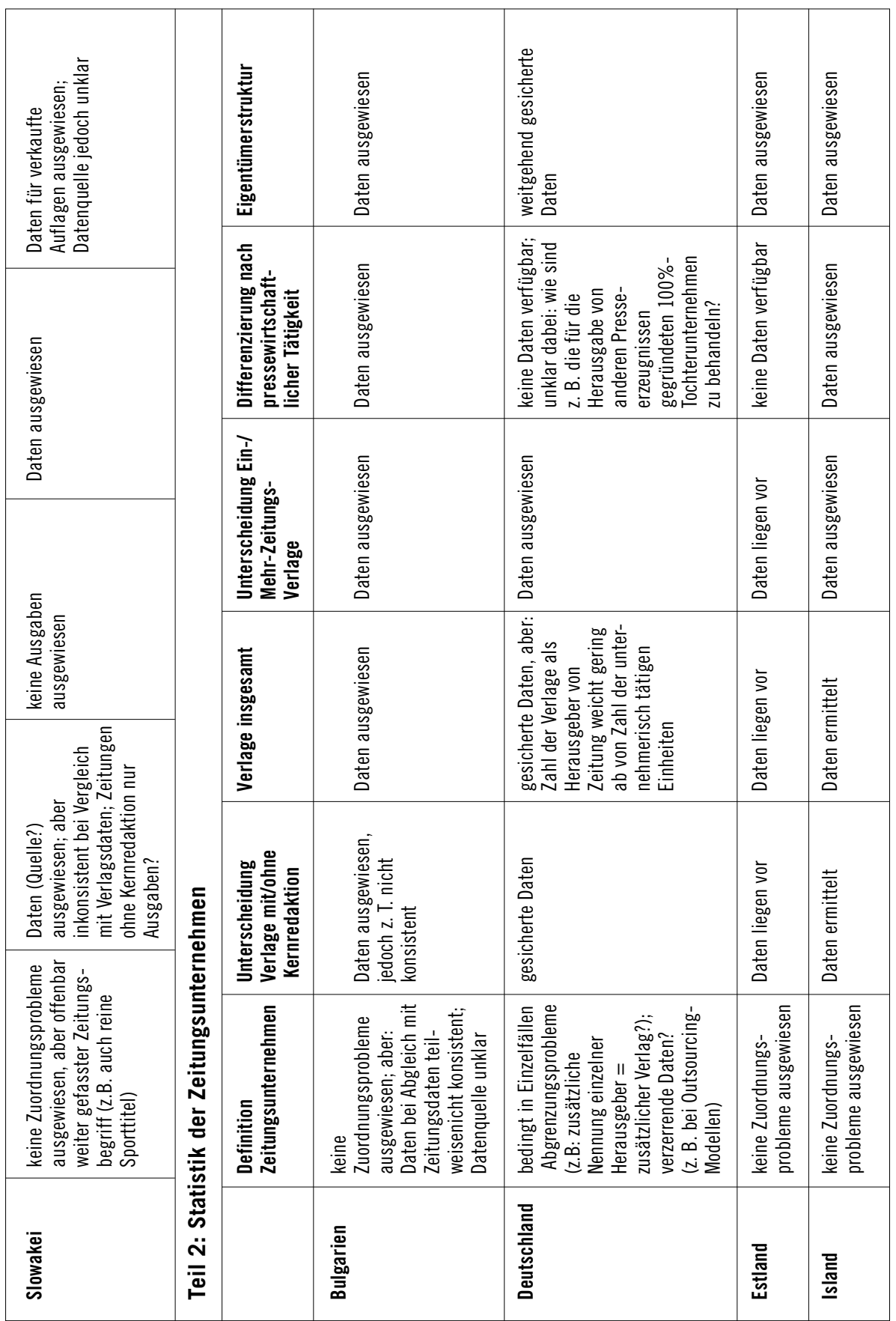




\begin{tabular}{|c|c|c|c|c|c|}
\hline 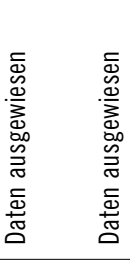 & 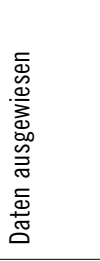 & 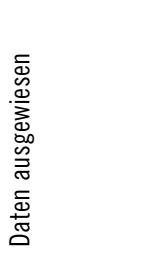 & 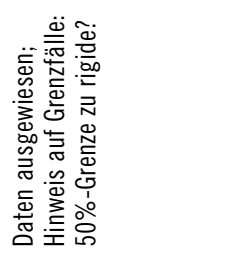 & 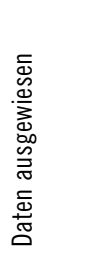 & 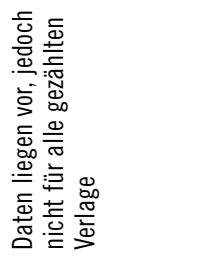 \\
\hline 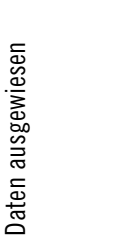 & 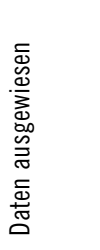 & 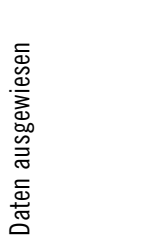 & 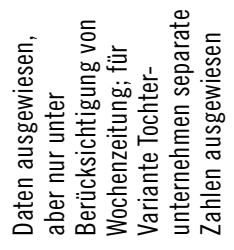 & 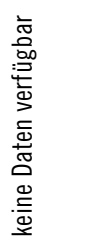 & 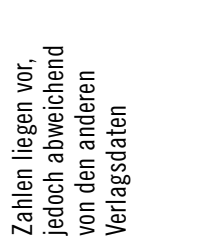 \\
\hline 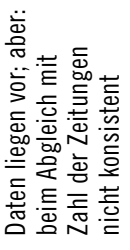 & 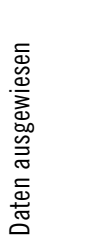 & 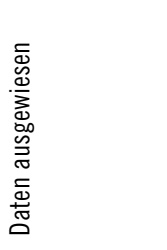 & 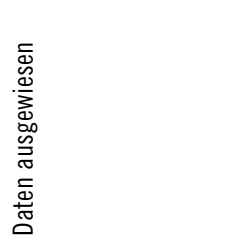 & 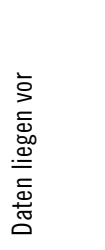 & 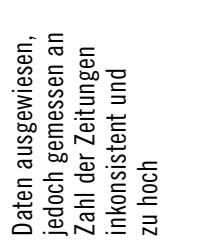 \\
\hline 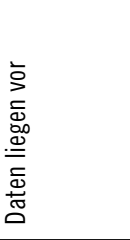 & 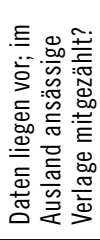 & 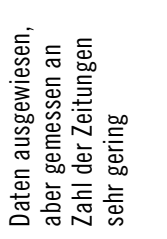 & 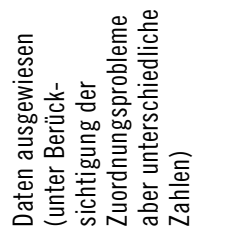 & 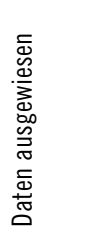 & 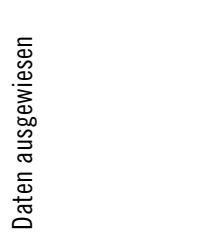 \\
\hline 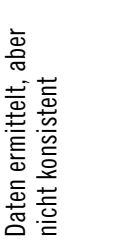 & 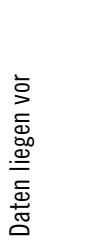 & 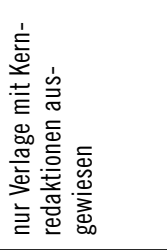 & 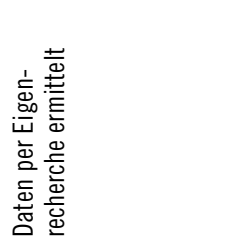 & 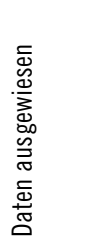 & 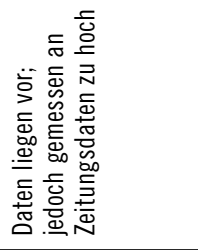 \\
\hline 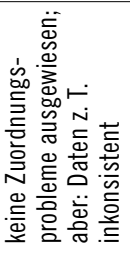 & 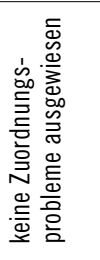 & 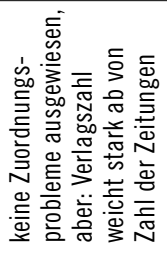 & 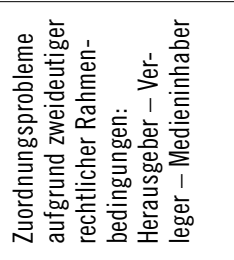 & 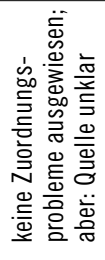 & 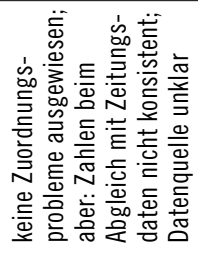 \\
\hline $\begin{array}{l}\text { Ф̄ } \\
\stackrel{\Xi}{\Xi}\end{array}$ & 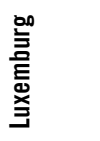 & 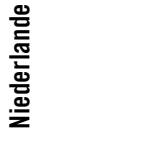 & 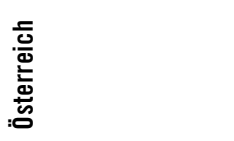 & $\frac{\bar{\sigma}}{0}$ & 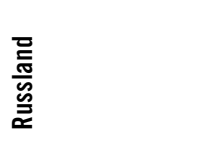 \\
\hline
\end{tabular}




\begin{tabular}{|c|c|c|c|c|c|c|}
\hline 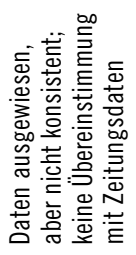 & 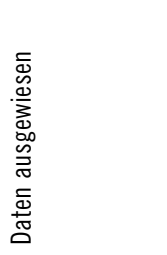 & \multirow{7}{*}{ 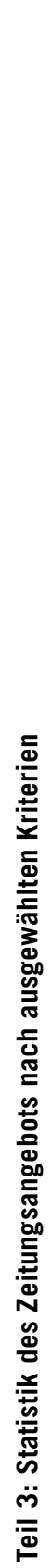 } & $\begin{array}{l}\text { 픔 } \\
\text { 혼 }\end{array}$ & 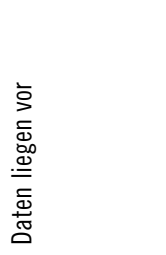 & 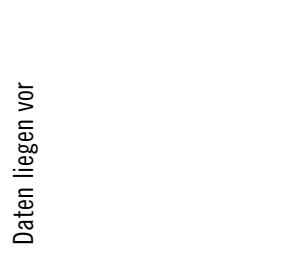 & 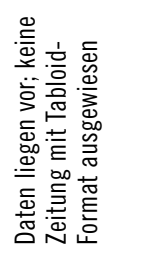 \\
\hline 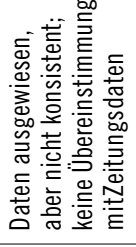 & 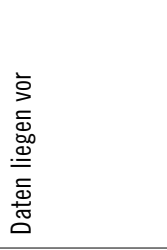 & & 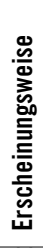 & 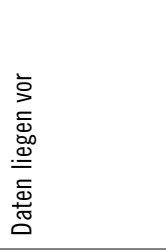 & 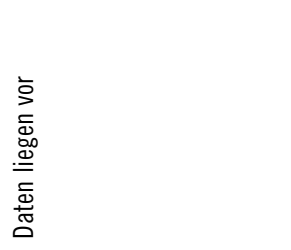 & 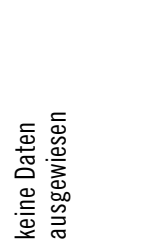 \\
\hline 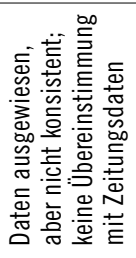 & 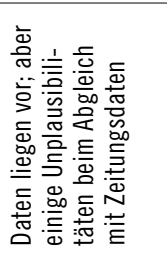 & & 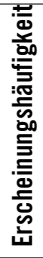 & 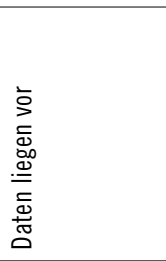 & 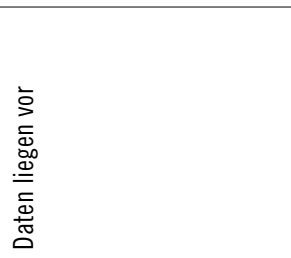 & 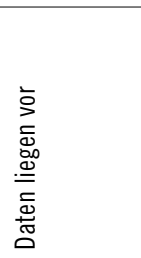 \\
\hline 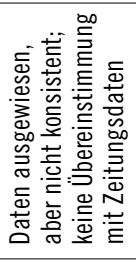 & 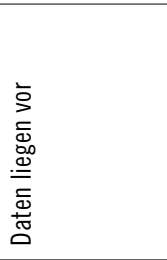 & & 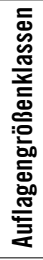 & 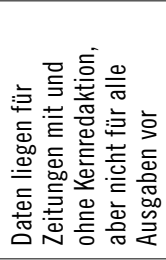 & 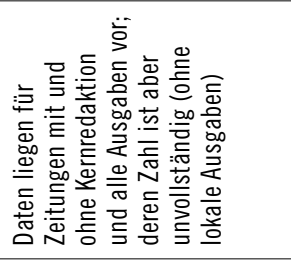 & 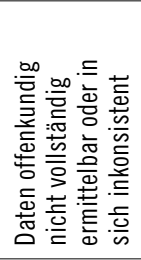 \\
\hline 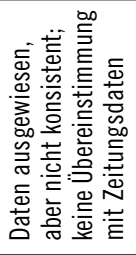 & 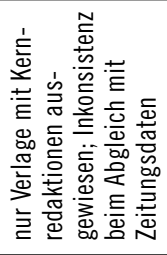 & & 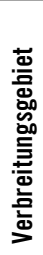 & 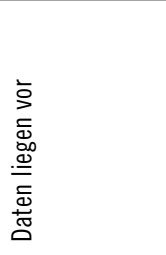 & 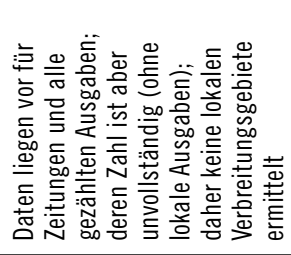 & 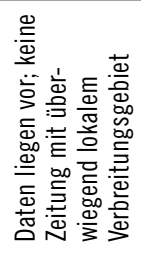 \\
\hline 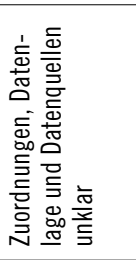 & 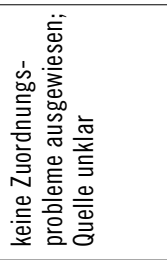 & & 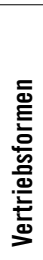 & 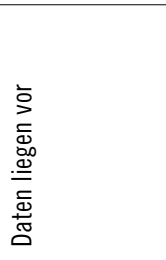 & 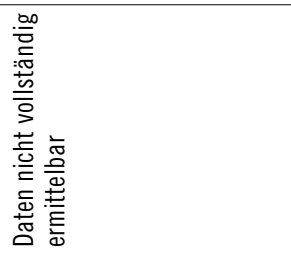 & 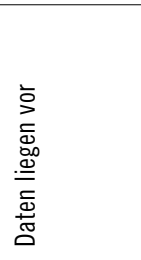 \\
\hline 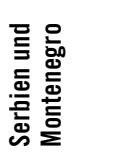 & 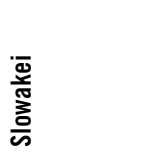 & & & 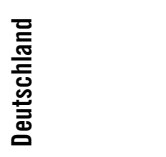 & 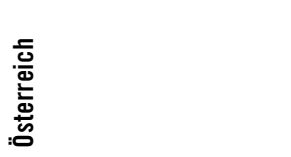 & $\begin{array}{l}\text { 을 } \\
\text { 혼 } \\
\text { 흘 } \\
\frac{1}{2}\end{array}$ \\
\hline
\end{tabular}




\begin{tabular}{|c|c|c|c|c|c|}
\hline 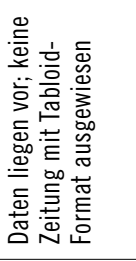 & 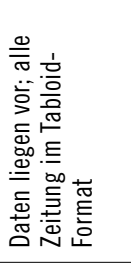 & 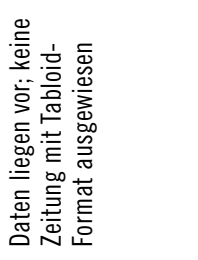 & 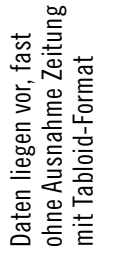 & 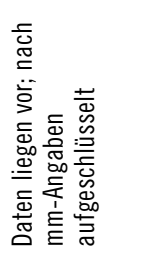 & 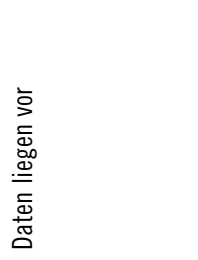 \\
\hline 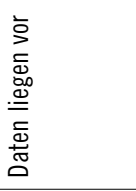 & 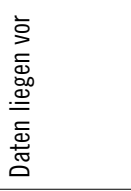 & 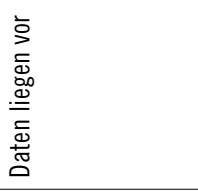 & 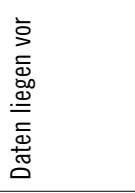 & 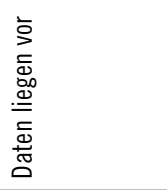 & 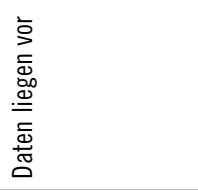 \\
\hline 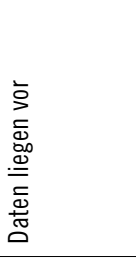 & 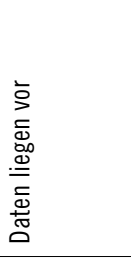 & 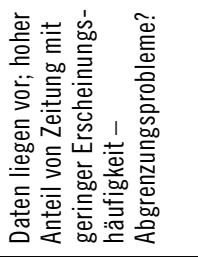 & 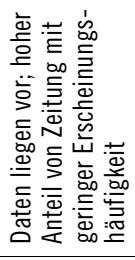 & 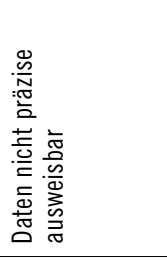 & 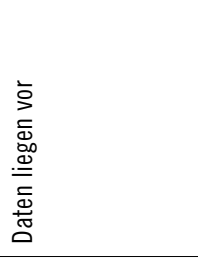 \\
\hline 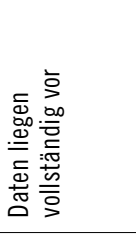 & 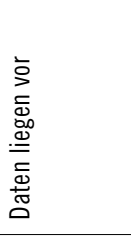 & 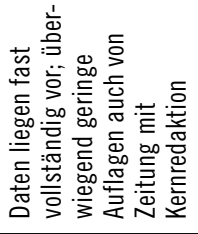 & 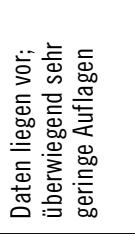 & 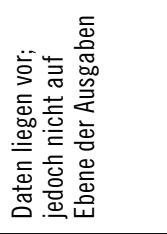 & 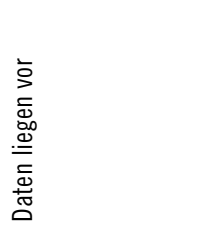 \\
\hline 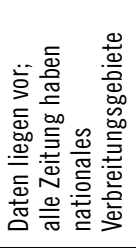 & 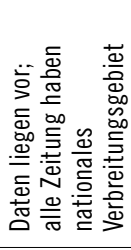 & 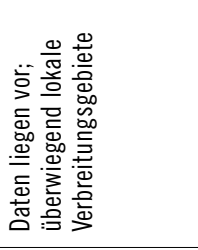 & 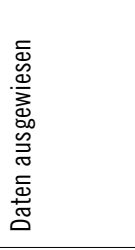 & 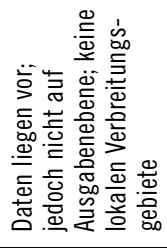 & 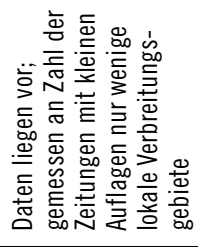 \\
\hline 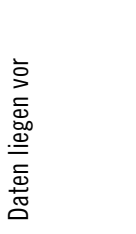 & 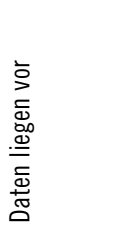 & 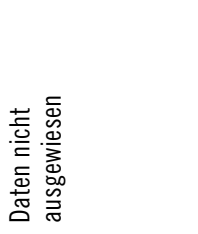 & 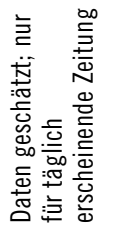 & 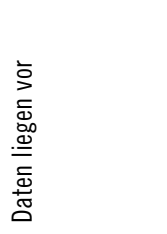 & 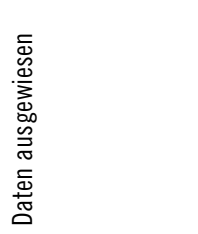 \\
\hline 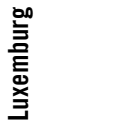 & $\begin{array}{l}\text { 믈 } \\
\text { 뜽 }\end{array}$ & 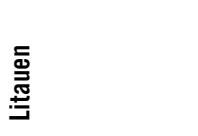 & $\begin{array}{l}\text { 플 } \\
\text { 产 } \\
\text { w }\end{array}$ & $\frac{\bar{\sigma}}{\overline{0}}$ & 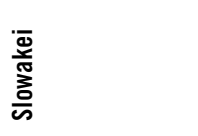 \\
\hline
\end{tabular}




\begin{tabular}{|c|c|c|c|c|c|c|c|}
\hline 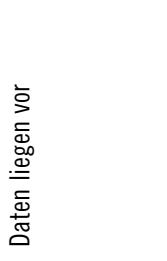 & 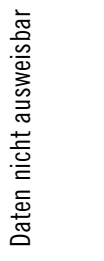 & 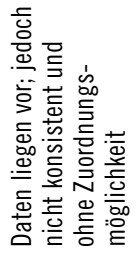 & 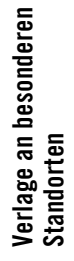 & 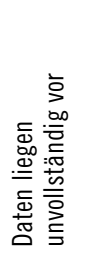 & 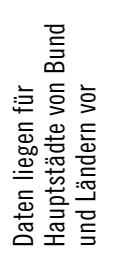 & 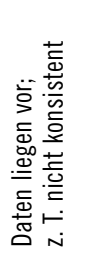 & 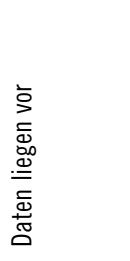 \\
\hline 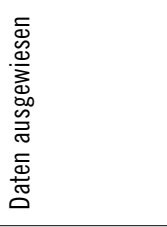 & 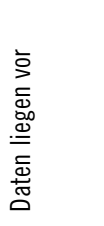 & 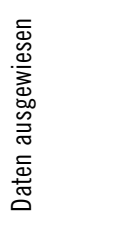 & 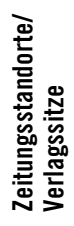 & 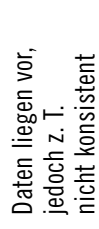 & 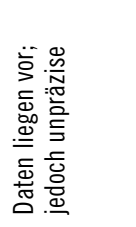 & 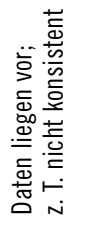 & 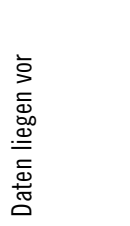 \\
\hline 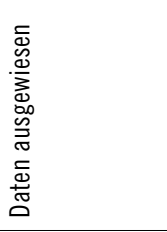 & 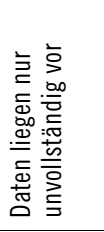 & 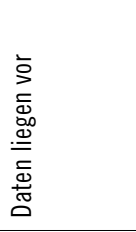 & 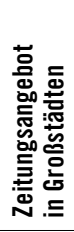 & 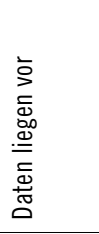 & 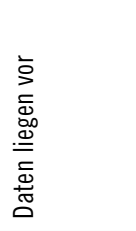 & 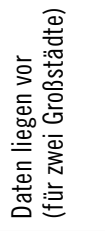 & 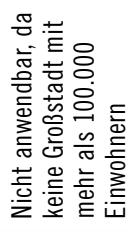 \\
\hline 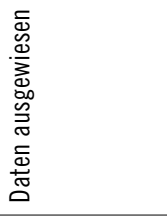 & 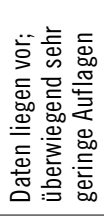 & 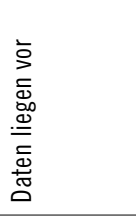 & 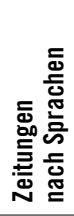 & 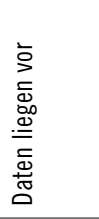 & 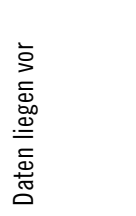 & 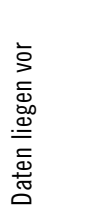 & 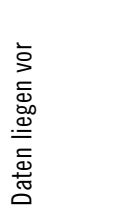 \\
\hline 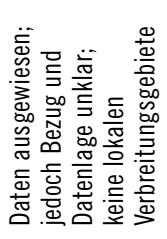 & 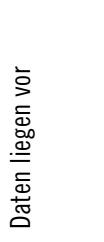 & 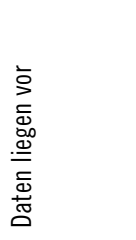 & 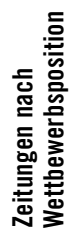 & 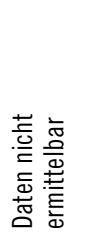 & 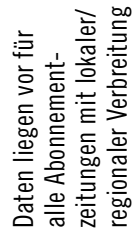 & 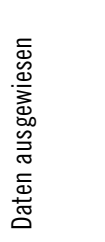 & 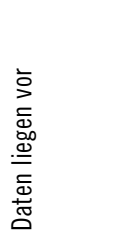 \\
\hline 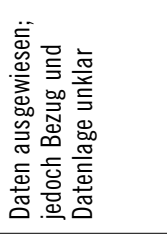 & 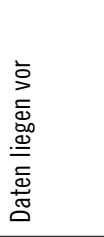 & 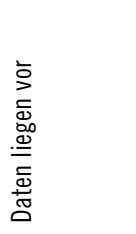 & 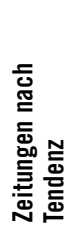 & 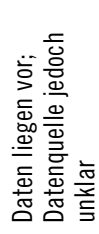 & 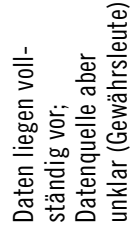 & 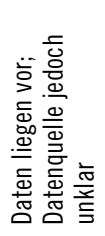 & 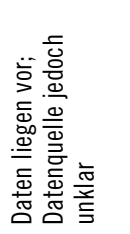 \\
\hline 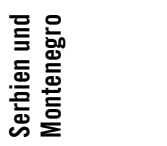 & $\begin{array}{l}\text { 흔 } \\
\text { 홈 } \\
\text { 잏 }\end{array}$ & 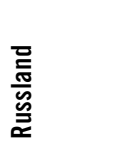 & & 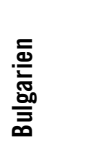 & 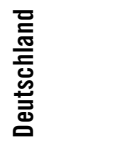 & 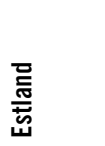 & $\begin{array}{l}\text { 트 } \\
\text { 产 }\end{array}$ \\
\hline
\end{tabular}




\begin{tabular}{|c|c|c|c|c|c|c|}
\hline 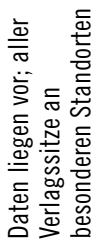 & 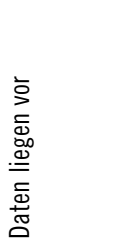 & 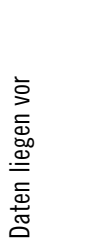 & 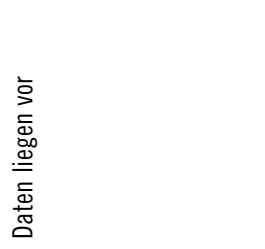 & 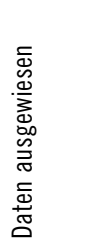 & 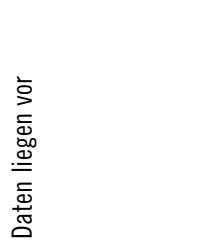 & 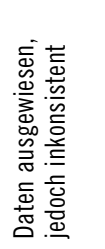 \\
\hline 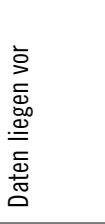 & 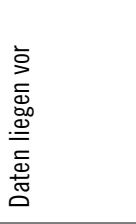 & 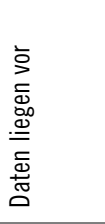 & 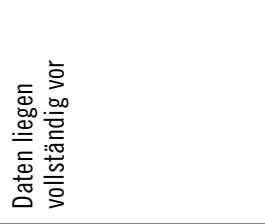 & 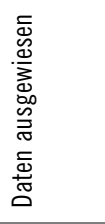 & 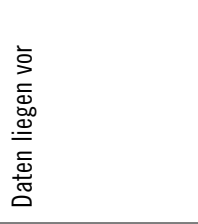 & 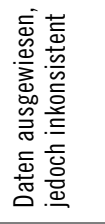 \\
\hline 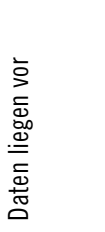 & 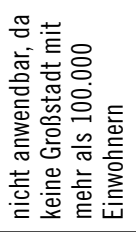 & 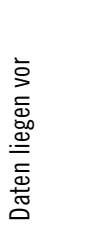 & 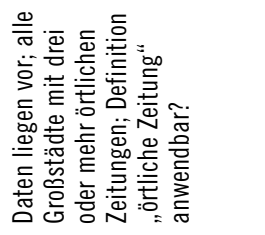 & 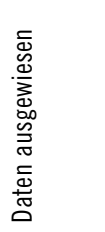 & 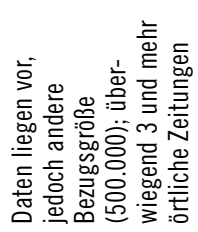 & 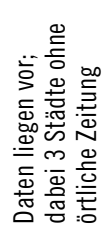 \\
\hline 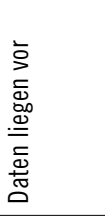 & 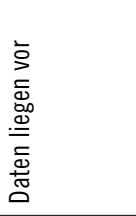 & 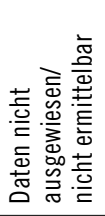 & 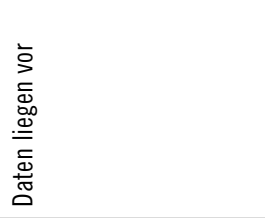 & 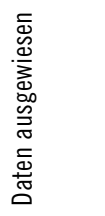 & 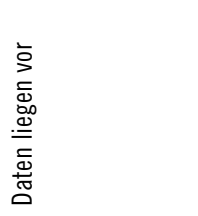 & 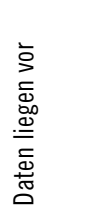 \\
\hline 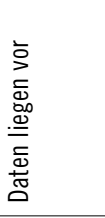 & 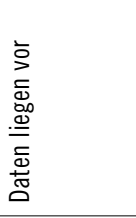 & 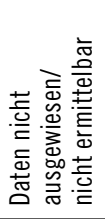 & 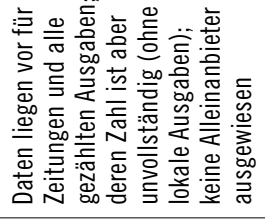 & 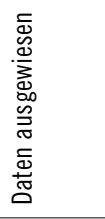 & 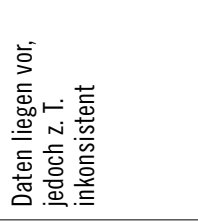 & 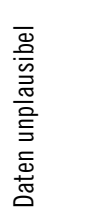 \\
\hline 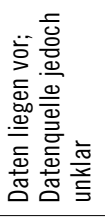 & 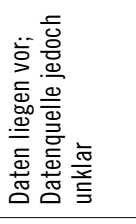 & 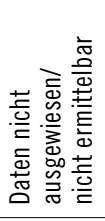 & 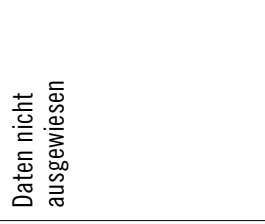 & 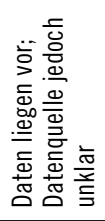 & 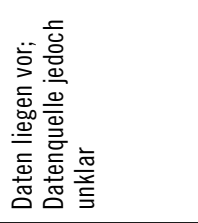 & 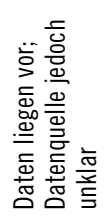 \\
\hline 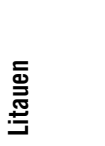 & 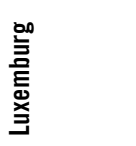 & $\begin{array}{l}\text { 을 } \\
\text { 혼 } \\
\text { 흘 } \\
\frac{0}{2}\end{array}$ & 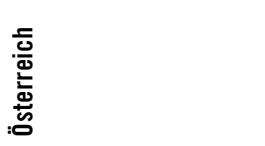 & $\frac{\bar{\sigma}}{\overline{0}}$ & 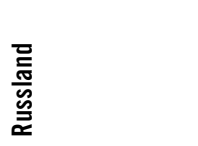 & 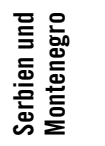 \\
\hline
\end{tabular}




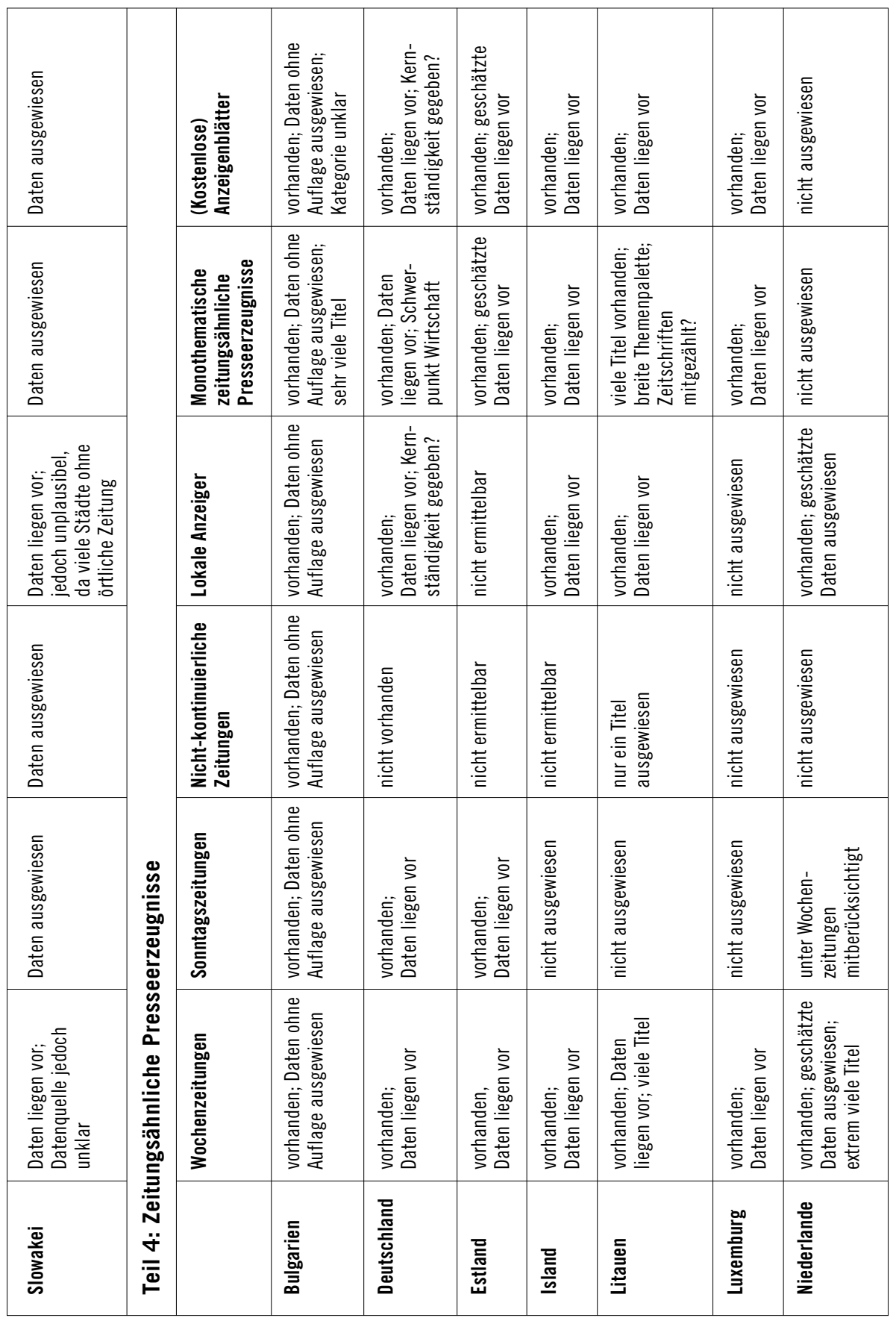




\begin{tabular}{|c|c|c|c|c|c|}
\hline 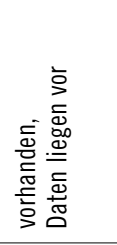 & 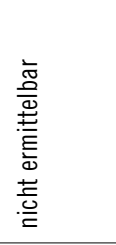 & 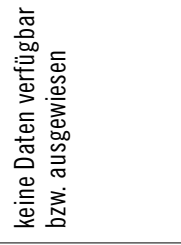 & 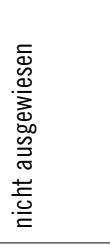 & 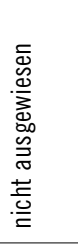 & \\
\hline 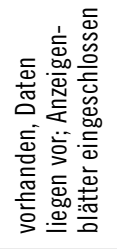 & 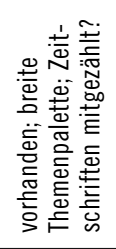 & 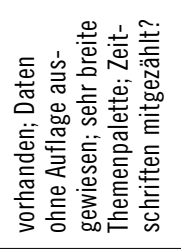 & 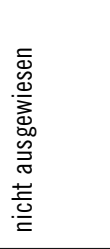 & 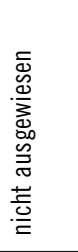 & . \\
\hline 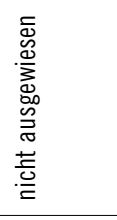 & 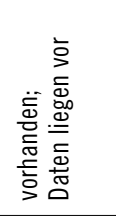 & 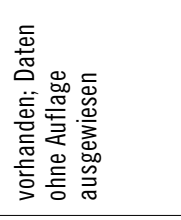 & 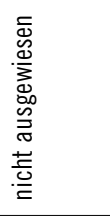 & 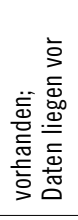 & 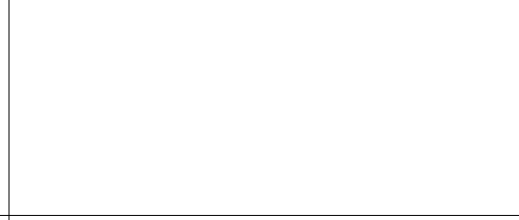 \\
\hline 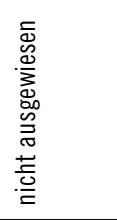 & 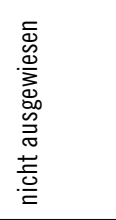 & 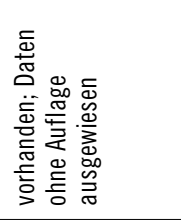 & 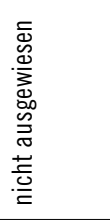 & 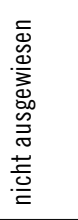 & . \\
\hline 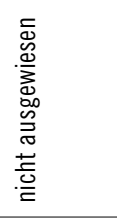 & 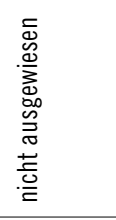 & 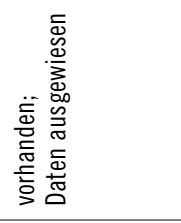 & 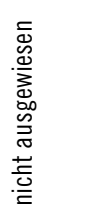 & 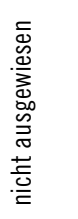 & . \\
\hline 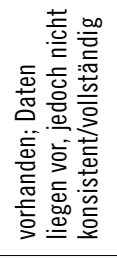 & 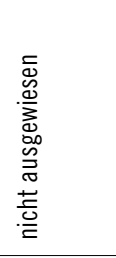 & 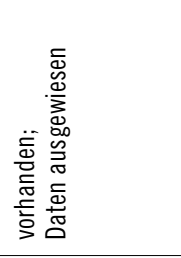 & 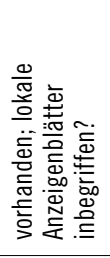 & 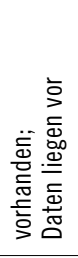 & \\
\hline 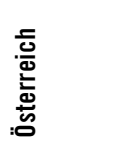 & $\frac{\text { ప }}{0}$ & 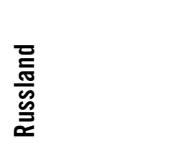 & 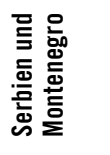 & $\frac{3}{\omega}$ & \\
\hline
\end{tabular}




\section{Praktikabilität der Kategorien und Datenlage: Länderprofile}

\section{Bulgarien}

- Kriterien für Pressetyp Zeitung nur bedingt übertragbar, der übliche Zeitungsbegriff ist weiter gefasst

- Differenzierung nach Zeitungen mit/ohne Kernredaktion nicht relevant

- Kategorie „Ausgaben“ anwendbar und statistisch erfassbar, <jedoch Vollständigkeit und Eindeutigkeit der Zuordnungen fraglich>

- Auflagenzahlen nicht für den gesamten Zeitungsmarkt auf gesicherter Datenbasis verfügbar

- Verlagsstruktur (hier vor allem der vorherrschende Einfluss ausländischer Unternehmen) auf Basis der vorgeschlagenen Kriterien nicht transparent und konsistent darstellbar

- Detailanalysen des Zeitungsangebots nur zum Teil möglich

- zeitungsähnliche Presseerzeugnisse teilweise statistisch erfasst

- <Datenverfügbarkeit und Datenquellen nicht geklärt>

\section{Deutschland}

- Restriktive Definition des Zeitungsbegriffs hat sich bewährt, bedingt jedoch in Einzelfällen strittige bzw. uneinheitliche Zuordnungen

- Differenzierung nach Zeitungen mit bzw. Zeitungen ohne Kernredaktion macht Ausmaß von publizistische Vielfalt transparent

- Kategorie „Ausgaben“ macht Ausmaß der (lokalen) Diversifizierung auf dem Zeitungsmarkt deutlich

- überwiegend verlässliche und kontrollierte Auflagenzahlen

- Verlagsstrukturen sind mit den verwendeten Kategorien umfassend abzubilden

- Ermittlung der Eigentumsstrukturen erfordert besondere Erhebungsinstrumente

- Vollständige Daten in den grundlegenden Kategorien ermöglichen Marktanalysen nach unterschiedlichen Kriterien (z. B. Auflagen-Größenklassen,Wettbewerbsverhältnisse, Umfang des Zeitungsangebots auf lokalen Märkten etc.)

- Datenlage und Datengüte für zeitungsähnliche Periodika nicht durchgängig zufrieden stellend

- Pressestatistik kann sich insgesamt auf breit angelegte und vielfältige Datenquellen stützen

\section{Estland}

- Vorgeschlagene Definition des Pressetyps Zeitung deckt sich nicht mit dem (weiter gefassten) Zeitungsbegriff dieses Landes

- Kriterium Kernredaktion vorhanden/nicht vorhanden ohne Relevanz 
- Kategorie „Ausgaben“ ebenfalls ohne praktische Bedeutung

- <Ausmaß der lokalen Ausrichtung der Zeitungen unklar bzw. teilweise widersprüchlich>

- <Basis und Zuverlässigkeit der Auflagendaten unklar>

- Verlagsstruktur weitgehend vollständig abbildbar

- Detailanalysen nach ausgewählten Kriterien möglich

- Statistik über zeitungsähnliche Periodika lückenhaft

- mehrere und unterschiedliche Datenquellen verfügbar

\section{Island}

- Zeitungsbegriff gemäß vorgeschlagener Definition ist praktikabel

- Differenzierung nach Zeitungen mit/ohne Kernredaktion sowie nach Ausgaben ohne Relevanz

- Zeitungs- und Verlagsstatistik wegen Überschaubarkeit und Homogenität der Marktstrukturen unproblematisch

- Detailanalysen nach spezifischen Kriterien problemlos realisierbar

- zeitungsähnliche Periodika statistisch gut erfasst

- singuläre, aber leistungsstarke Datenbasis

\section{Litauen}

- Praktikabilität der Definition des Zeitungsbegriffs wie auch der zentralen pressestatistischen Kategorien grundsätzlich gegeben, aber:

- Befunde deuten auf Plausibilitätsprobleme hin

- gemessen an Einwohnerzahl ungewöhnlich große Zahl an Zeitungen

- fast ausschließlich eigenständige Kernredaktionen

- weitgehend ohne Ausgabendifferenzierung

- im Durchschnitt sehr geringe Auflagen

- <Zeitungs- und Verlagsstatistik sind teilweise nicht in Einklang zu bringen>

- Detailanalysen des Zeitungsmarktes lassen sich vornehmen

- für die verschiedenen zeitungsähnlichen Periodika werden zum Teil Zuordnungsprobleme erkennbar

- statistische Datenbasis für viele Bereiche ungeklärt

\section{Luxemburg}

- definierte Kriterien für Pressetyp Zeitung sind problemlos übertragbar

- Unterscheidung nach Kriterium Kernredaktion hat für Zeitungsmarkt keine Bedeutung

- Differenzierung nach lokalen/regionalen Ausgaben nicht relevant

- <Ausgabendifferenzierung nach Sprachgruppen gegeben?>

- statistische Zuordnung einzelner Ausgaben von ansonsten im Ausland ver- 
legten Zeitungen problematisch

- Verlagsstatistik gemäß vorgeschlagener Definition weitgehend unproblematisch

- Kontrollierte Auflagendaten liegen vor

- Detailanalysen des Zeitungsmarktes sind realisierbar

- Zuordnungs- und Abgrenzungsprobleme bei zeitungsähnlichen Periodika

- breite, aber nur zum Teil gesicherte Datenbasis

\section{Niederlande}

- restriktive Definition des Zeitungsbegriffs wird den Besonderheiten des Pressemarktes unter Umständen nicht gerecht

- geringe Zahl von Zeitungen bei zugleich sehr hoher Zahl von zeitungsähnlichen Produkten, vor allem Wochenzeitungen und lokale Anzeigeblättern

- Differenzierung nach Zeitungen mit/ohne Kernredaktion nicht relevant

- <Kategorie „Ausgabe“ möglicherweise nur lückenhaft ermittelbar;> keine Zeitungen mit primär lokaler Ausrichtung

- gemessen an der Zahl der Zeitungen nur sehr wenige Verlage - und noch weniger Mehr-Zeitungs-Verlage - nachgewiesen; Definition für Zeitungsunternehmen praktikabel?

- Detailanalysen des Zeitungsmarktes nach bestimmten Kriterien sind nur zum Teil möglich

- Transparenz für einige zeitungsähnliche Periodika nur in Ansätzen, für andere überhaupt nicht gegeben

- <insgesamt nur spärliche Datenbasis>

\section{Österreich}

- Zeitungsbegriff gemäß der vorgegebenen Definition ist grundsätzlich anwendbar

- Differenzierung nach Kernredaktion vorhanden/nicht vorhanden kaum relevant

- <Kategorie „Ausgabe“ nur national und regional ermittelbar, daher lokale Verankerung der Zeitungen kaum darzustellen>

- <Ermittlung verlässlicher bzw. kontrollierter Auflagendaten nur lückenhaft möglich>

- Verlags- und Eigentumsstrukturen teilweise nicht eindeutig, aber insgesamt hohes Konzentrationsniveau feststellbar

- für die verschiedenen Typen von zeitungsähnlichen Periodika werden zum Teil Abgrenzungsprobleme und eine insgesamt lückenhafte Datenbasis sichtbar

- Datenbasis insgesamt vielfältig, aber nicht durchgängig konsistent 


\section{Polen}

- Definitionskriterien für Pressetyp Zeitung praktikabel

- Differenzierung nach Kriterium Kernredaktion vorhanden/nicht vorhanden nahezu ohne Bedeutung

- statistische Erfassung von Ausgaben ist erfolgt, Vollständigkeit und Konsistenz der Daten teilweise jedoch unklar

- Auflagenzahlen vorliegend, Datenbezug und Datenquelle jedoch unklar

- Verlagsstrukturen nahezu umfassend abbildbar

- gute, allerdings nicht vollständige Markttransparenz auch für verschiedene Detailanalysen gegeben

- Statistik für zeitungsähnliche Periodika sehr lückenhaft und mit erkennbaren Zuordnungs- bzw. Abgrenzungsproblemen

- verschiedene Datenquellen auswertbar, jedoch Datenbasis nicht für alle Bereiche geklärt

\section{Russland}

- Definitionskriterien für Pressetyp Zeitung sind praktikabel

- Unterscheidung nach dem Kriterium Kernredaktion vorhanden/nicht vorhanden ist umsetzbar - bei allerdings nicht durchgängig konsistenten Befunden

- Kategorie „Ausgabe“ ist statistisch darstellbar; Zuordnungskriterien und Vollständigkeit jedoch nicht eindeutig

- Auflagenzahlen nur bezogen auf Druckauflage und auf ungesicherter bzw. nicht geklärter Datenbasis verfügbar

- Verlagsstatistik nicht eindeutig

- Detailanalysen des Zeitungsangebots nach ausgewählten Kriterien umfassend möglich, aber:

- zahlreiche spezifische Probleme und Besonderheiten des russischen Zeitungsmarktes statistisch kaum abbildbar

- Daten für zeitungsähnliche Periodika verfügbar, aber Unklarheit über Vollständigkeit und teilweise über die Zuordnungskriterien

- Datenquellen und Datenbasis in weiten Bereichen ungeklärt

\section{Serbien und Montenegro}

- <Übertragbarkeit der Definition des Pressetyps Zeitung problematisch, daher Zuordnungs- und Zählkriterien nicht eindeutig>

- Differenzierung nach Kernredaktion vorhanden/nicht vorhanden sowie nach der Kategorie „Ausgabe“ nicht darstellbar oder von keiner praktischen Relevanz

- Verlagsstrukturen mit den vorgeschlagenen Kriterien nicht präzise und konsistent darstellbar 
- Daten über Zeitungsauflagen nur auf Basis von Schätzungen oder Eigenangaben der Verlage verfügbar

- Aussagekraft von Detailanalysen des Zeitungsmarktes aufgrund der nur spärlichen Datenbasis gering

- Statistik für zeitungsähnliche Presseerzeugnisse sehr lückenhaft

- Datenverfügbarkeit/Datenbasis offenbar gering bzw. ungeklärt

\section{Slowakei}

- <Übertragbarkeit der vorgeschlagenen Zeitungsdefinition scheint problematisch>

- Differenzierung nach den Kategorien „Kernredaktion“ und „Ausgaben“ nicht relevant oder nicht möglich

- Auflagenzahlen vorliegend, Aussagekraft <und Datenquelle> jedoch unklar

- Verlagsstrukturen gemäß vorgeschlagener Kriterien darstellbar

- Detailanalyse des Zeitungsmarktes nach besonderen Kriterien möglich; Befunde jedoch teilweise nicht konsistent bzw. mit Plausibilitätsproblemen

- Zeitungsähnlichen Presseerzeugnisse statistisch erfasst; Zuordnungsprobleme sind allerdings zu vermuten

- <Datenbasis und Datenquellen insgesamt ungeklärt> 